\title{
The journey from self-care to GP care: a qualitative interview study of women presenting with symptoms of urinary tract infection
}

\author{
Geraldine M Leydon, Sheila Turner, Helen Smith and Paul Little
} on behalf of the UTIS team

\begin{abstract}
Background

Urinary tract infection (UTI) is one of the commonest acute infections presenting to primary care. Little is known of women's experiences of UTI; self-care strategies and key triggers for their consulting behaviour are also little known.

Aim

To explore women's experiences of self-care and their journey to GP care, when faced with symptoms of a UTI.

Design of study

Qualitative semi-structured interview study with women recruited to a larger UK trial of different management strategies for UTI.

Setting

General practices across four counties in southern England.

\section{Method}

Twenty-one women were interviewed about the experiences they had prior to their GP visit, self-care strategies, and triggers for help seeking. Interviews were analysed thematically, using principles of analytic induction.
\end{abstract}

\section{Results}

Women reported a process of evaluation, monitoring, re-evaluation, and, finally, consulting in order to meet their needs. Four key triggers for consulting were identified: failure to alleviate symptoms through selfcare; symptom duration and escalation; impeding normal functioning and the fulfilment of social roles; and concern that it may be or become a serious illness.

\section{Conclusion}

Although UTI is often self-limiting, when taking patient histories and formulating their management strategies clinicians need to take into account women's often painful experience, their efforts to resolve symptoms prior to consulting, and their fears that the symptoms may indicate something more serious than a UTI.

Keywords

antibiotics; consultation; urinary tract infection.

\section{INTRODUCTION}

Acute urinary tract infection (UTI) is one of the commonest acute bacterial infections among women. ${ }^{1,2}$ Little is known of women's self-care strategies and core triggers for seeking professional help. An improved understanding of the journey to the doctor when faced with UTI symptoms can aid theoretical understanding of women's self-care and health-seeking behaviour. This understanding may help to improve negotiation of management strategies in consultations between GPs and patients and optimise the balance between primary care and selfcare for UTI. ${ }^{3,4}$

\section{METHOD}

\section{Participants and procedure}

Patients were drawn from practices across the four counties of Berkshire (Reading), Wiltshire (Salisbury), Hampshire (Romsey, Portsmouth, Waterlooville, Havant), and Dorset (Dorchester). To be eligible for inclusion, patients had to: be participants in a randomised controlled trial of different management strategies; and have consented to participate in a single

GM Leydon, BA, MA, PhD, DipPH, senior researcher fellow; P Little, MD, BA, MSc, MRCP, FRCGP, DLSHTM, professor of primary care research, Primary Medical Care, Community Clinical Sciences Division, University of Southampton School of Medicine. S Turner, BSc, PhD, NIHR senior research fellow, NIHR Public Health Research Programme, Coordinating Centre for Health Technology Assessment, Southampton. H Smith, MSc, DM, MRCGP, FFPHM, professor of primary care, Brighton \& Sussex Medical School, Brighton.

Address for correspondence

Geraldine M Leydon, Primary Medical Care, Community Clinical Sciences Division, University of Southampton School of Medicine, Aldermoor Health Centre, Southampton, SO16 5ST. E-mail: gerry@soton.ac.uk

Submitted: 17 October 2008; Editor's response: 10 December 2008; final acceptance: 26 January 2009.

(C)British Journal of General Practice

This is a full-length article of an abridged version published in print and originally published online first on 11 June 2009. Cite this version as: Br J Gen Pract 2009 DOI: 10.3399/bjgp09X453459 (abridged text, in print: Br J Gen Pract 2009; 59: 490-495). 
face-to-face interview. Within the GP consultation trial, participants were randomised to one of five management groups (see Box 1 for more details):

- empiric antibiotic treatment;

- empiric delayed antibiotics;

- antibiotic targeted by two or more features: cloudy urine, offensive-smelling urine, moderately severe dysuria, or nocturia;

- by dipstick algorithm (nitrites, or leucocytes and a trace of blood); or

- mid-stream urine (MSU; symptomatic treatment until MSU results available, antibiotics targeted according to MSU results).

Interviews were conducted in women's homes and lasted an average of 1 hour, they were audiotaped and transcribed verbatim by a professional freelance transcriptionist, and a sample checked against the recorded interview to ensure transcription accuracy.

\section{The interviews}

Qualitative interviews provided the optimal method for collecting retrospective experiences and views on this relatively unexplored and personal condition. A semistructured topic guide ensured that potentially important topics derived from a previous study were covered in each interview, ${ }^{5}$ but retained the flexibility for participants to raise issues germane to them (Appendix 1). The interviews were designed to elicit interviewees' journey to their GP, including self-care strategies and eventual triggers to consult.

\section{Analysis}

Using principles of a modified analytic induction method, thematic analysis was conducted in an iterative manner, moving back and forth between interview transcripts, analytic memos, notes about process, and the research literature. Vertical and horizontal familiarisation of the interviews was aided by producing summaries of each. ${ }^{6-8}$ Following repeated readings (while listening to taped interviews), an early coding framework was developed based on five transcripts, and this was iteratively developed during analysis of the full data corpus. 'Crude counts' of observations/themes provided an indication of the frequency of themes. ${ }^{6}$ In a sample of transcribed interviews, the validity of the codes were independently checked and the accuracy/reliability of their application to the transcribed data. The consistency of coding was also checked by iteratively revisiting (and re-analysing) transcripts at different times. Codes were iteratively developed by all authors, and eventually all data organised and codes merged to generate themes that captured the range of experiences and views reported.

\section{How this fits in}

Urinary tract infection (UTI) is largely self-limiting, yet the majority of women who attend a GP with UTI symptoms will be prescribed antibiotic medication. Insufficient knowledge exists regarding women's self-care prior to a GP visit and the core triggers for seeking help when faced with UTI symptoms. The majority of women in this study attempted some form of self-care; their journeys to the consulting room were highly evaluated and were often protracted and uncomfortable. Blood in the urine was cause for concern, and seeking reassurance was a major trigger for attending a GP. Prior knowledge of these experiences, fears, and expectations can help clinicians sensitively elicit patient histories and communicate their preferred management strategies, without risking women feeling their attendance is somehow misplaced or not valid. This study confirms the continued relevance of existing theories about illness behaviour and help seeking in the previously neglected area of UTI.

Exemplary quotations from the transcribed interviews are used to illustrate key themes (after each quotation bracketed information describes which interview transcript [interview 1-20] has been quoted and the line number[s] of the extracted quotation [for example, 33-36]).

\section{Box 1. Management strategies used in the main trial.}

The five management strategies used in the main trial represent common approaches in general practice.

- Empiric antibiotic treatment. This is the most common strategy in practice and was used as the control group. Patients were prescribed an antibiotic (trimethoprim $200 \mathrm{mg}$ bd) for 3 days. If patients were allergic to trimethoprim they were offered an alternative (cefaclor or cefalexin) since this is not a trial of antibiotics only but a trial of management and advice strategies.

- Empiric delayed antibiotics. All patients were advised to drink plenty, and offered a delayed antibiotic prescription to be used if symptoms did not start to improve after 48 hours (doctors were asked to leave a prescription at the front desk for patients to collect as necessary, or could negotiate with the patient if they wanted to take the prescription away). The rationale for this group is that $40 \%$ of patients with suspected UTI do not have infection, and even in those with laboratory-diagnosed infections, the illness is likely to be self-limiting.

- Symptom score. Patients who had two or more of the following four features (urine cloudy on examination, urine offensive smell on examination, patient report of moderately severe dysuria, patient report of moderately severe nocturia), were offered immediate antibiotics. From the previous study, the sensitivity of this symptom score approach was estimated as $68 \%,{ }^{16}$ so patients without two or more features were also offered a delayed antibiotic prescription to use if their symptoms were not settling after a few days.

- Dipstick. Patients who had either nitrites, or leucocytes and a trace of blood, were offered antibiotics initially. Patients not fulfilling the above criteria (which this study estimates had a sensitivity of $71 \%$ ) were offered a delayed antibiotic prescription to use if their symptoms were not settling after a few days.

- Mid-stream urine (MSU). This was the only group in which an MSU was done routinely. Patients were offered symptomatic treatment until the results of the MSU were known. This is the 'reference' method of diagnosing infection and of targeting antibiotic use. 


\begin{tabular}{|c|c|}
\hline Problem or complaint & Incidence, $n$ \\
\hline \multicolumn{2}{|l|}{ Physical } \\
\hline Urinary frequency & 8 \\
\hline Very painful/severe/bad & 7 \\
\hline Bleeding & 6 \\
\hline Cold/flu-like symptoms/temperature & 4 \\
\hline Backache/pain & 3 \\
\hline Stinging/burning/stabbing & 3 \\
\hline Pains/balloons in tummy & 3 \\
\hline Uncomfortable & 3 \\
\hline Poor concentration & 2 \\
\hline Pain when urinating & 2 \\
\hline Smelly urine & 2 \\
\hline Tired/exhausted & 2 \\
\hline Sleepless & 1 \\
\hline Hot sensation in bladder & 1 \\
\hline Pain worsening & 1 \\
\hline \multicolumn{2}{|l|}{ General/emotional/functional } \\
\hline Generally unwell/lousy/poorly & 6 \\
\hline Normal duties disrupted/debilitating & 3 \\
\hline
\end{tabular}

\section{RESULTS}

\section{Participants}

Thirty-three women were approached to take part in the interview study. Twenty-seven agreed and 21 (aged 21-64 years [median age 40 years]) were interviewed before data saturation was reached (one tape failure meant that 20 interviews were analysed). Reasons for refusal included being too busy or unavailable for interview.

The characteristics of participating women in this study were similar to the trial cohort: married $65 \%$ versus $73 \%$ respectively; past cystitis: $88 \%$ versus $85 \%$; number of concurrent medical problems: 3.0 versus 2.6; age leaving education: 17.6 versus 17.6 years; and severity of urinary frequency symptoms at baseline mean: 3.5 versus 3.5. Seven women were in the symptoms score group, nine in the empirical delayed group, and two each in symptoms score and MSU groups. Women were selected for interview who

\begin{tabular}{|c|c|}
\hline $\begin{array}{l}\text { Time waited prior } \\
\text { to GP visit }\end{array}$ & $\begin{array}{c}\text { Interviewees' } \\
\text { who reported wait }\end{array}$ \\
\hline 1 night and 1 day & 18 \\
\hline 2-3 days & $8,11-13,15,17$ \\
\hline 4 days & 6 \\
\hline 7 days & $7,9,14,16,20$ \\
\hline 10 days & 4 \\
\hline 3 weeks & 2,19 \\
\hline 4 weeks & 1 \\
\hline Unclear & $3,5,10$ \\
\hline
\end{tabular}

had been asked to delay taking antibiotics, in order to obtain their views on the delay strategy. This would not apply to women in the immediate empiric antibiotic group.

The interviews took place in the context of a trial of GP management, and consequently excluded women who did not consult their GP. The nonconsulters may have included women whose selfcare was effective, some whose symptoms were not severe or prolonged, and possibly some women who wished to avoid antibiotics. By the time of the interviews, most of the participants were no longer experiencing symptoms and reported being free of infection.

\section{Patient experiences of UTI and the journey to the GP}

In their interviews women recounted their experiences from symptom onset and their attempts to self-manage, through to their final decision to attend a GP. The duration and type of symptoms experienced prior to consulting are summarised in Tables 1 and 2.

Interviewees reported a process of evaluation whereby they appraised their symptoms and decided upon a diagnosis, which enabled them to target selfcare. The initiation of self-care was followed by a period of monitoring which led most to conclude that self-care had in fact failed to work fully or in a timely fashion (this was true for all but two women who had decided from the outset that they required antibiotic medication). Failed self-care led women to re-evaluate their needs and consider whether their symptoms had begun to interfere with work and leisure activities, and for many this also included evaluating their fears that their experience may indicate something more serious than a UTI. It was at this juncture that women reported having to meet their re-evaluated needs by seeking GP help. This paper focuses on four common triggers to this process.

\section{Triggers for seeking GP care}

Failure to alleviate symptoms through lay remedies. Failure to alleviate their symptoms through self-care was by far the commonest cited rationale for consulting (interviews 2, 7-12, 16, and 18):

'I tried self-medication which didn't work ...' (interview 10: 5-6)

'... Prior to [the GP visit] I had taken, done the usual thing, drink plenty of fluid, that didn't work, so l'd been into the chemist and bought one of the overthe-counter remedies. That didn't work, so that's when I went to the doctor in the end ...' (interview 16: 8-11) 
It is worth noting that when describing their pathways to the doctor, interviewees went to considerable (narrative) lengths to portray themselves as responsible consumers of health services. For example, in the following exemplary extract, the interviewee's language works to portray her as having acted reasonably when faced with illness:

... I just drank lots and lots of fluids and ... cranberry juice ... but it wasn't making any difference at all ... so I went to the doctor ...'

and later:

'I think I must have gone through ... there was at least 3 days where all I drank was cranberry juice and it made no difference ... never been [to doctors for a UTI] before but I just thought at this point enough is enough.' (interview 14: 53-65; author's emphasis)

Sometimes the corollary of a 'failed' attempt to selfcare was the simple 'dragging on' or eventual escalation of symptoms.

\section{Symptom type, duration, or escalation}

Some described how their decision to seek medical help resulted from this episode being qualitatively different from previous experiences or that their bodies were 'not right':

'... I think I said to the doctor at the time y'know it wasn't horrendous it was just niggling and I just knew it wasn't right' (interview 2: 88-90)

'This time was different.' (interview 12: 8)

'I woke up with really bad stabbing pains in my back, um, and it just felt different ... so I went to the doctor. It didn't feel the same ...' (interview 8: 18-19)

Relative to previous episodes, the index episodes were sometimes experienced as out of the ordinary or 'not normal' in terms of duration:

I've had it a couple of times before and it's gone within a couple of days, but this was just dragging on ...' (interview 12: 32-36)

'Normally if it's going to shift, it'll shift quite quickly, you know, you get some sort of feeling that it's going to be moving on and you're going to be OK. But this time I didn't so I thought, you know, I'm going to the doctor ...' (interview 10: 9-12)
And, sometimes in terms of severity:

I've had them before but never as bad as [this one] ...' (interview 14: 35)

Most participants explicitly noted the escalation of symptoms as a key trigger to consult:

'It started mild, then got worse so that's why I went to the doctor.' (interview 18: 14)

Some mentioned their concern that worsening symptoms may indicate something more serious necessitating medical attention. In such circumstances, interviewees' reports oriented to help seeking as the most reasonable course of action:

'It wasn't getting better it was getting steadily worse and I thought "well I want to nip this", because you never know really, I had a pain in my back as well ... and I wasn't sure if it was y'know kidneys ... so I thought I better go then.' (interview 5: 141-148)

While there was a sense that participants sought medical help when they just could no longer tolerate pain, the situation was more complex than a 'single question of pain'. ${ }^{9}$

\section{Impeding normal functioning and the fulfilment of social roles}

As described in Zola's work on pathways to the doctor, ${ }^{9}$ it was clear that participants did not appeal to symptoms alone, but the perceived implications of the symptoms were also a driver for help seeking. One such implication was a 'perceived interference with vocational or physical activity'. Some of the participants referred to their child care duties:

'... I started drinking cranberry juice, and I drink a lot of water at work anyway, but it just didn't get any better, and having children I didn't want to feel any more poorly than I was feeling ... so I decided to go to the doctor ...' (interview 9: 14-19)

Most, however, appealed to a reason that is 'straight out of the protestant ethic' or to a duty to stay well in order to fulfil their roles in the community. ${ }^{9}$ Indeed, participants commonly invoked a classic Parsonian argument for help seeking, ${ }^{10}$ that is, they not only sought a speedy recovery, but they did so in order to enable the fulfilment of their social roles:

'... it was making it difficult to work, um, and I was teaching classes as well.' (interview 8: 19-20) 
'I was in pain quite a lot and I couldn't just get on with my normal day-to-day things ... I went to work but it was very difficult. So I went to the doctor's ...' (interview 12: 11)

Pain combined with interruption to everyday life provided a powerful rationale for help seeking:

'It just got worse and worse and it got to the stage where I couldn't go to work and I was just in agony.' (interview 11: 28)

Work responsibilities and failure to fulfil them was the dominant precursor to help seeking, with only one interviewee reporting that the frequent need to void hampered her leisure activities as well as her work (interview 14: 202-211).

Participants also described the impact of their symptoms on life in general:

I was just to the point, you know, it, it stops life doesn't it ... it stops normal living and that's when I went.' (interview 17: 6-10)

'Debilitating' symptoms were described as eventually leading to a break point where 'enough was enough', and the trigger for help seeking was activated:

'... it sort of became more and more debilitating and it was realising how debilitating it could be ... the effects of having it became ... quite big ... it was affecting how I was feeling generally, like emotionally and physically.' (interview 14: 203-207)

\section{Concern that it might be a serious illness or become serious}

An additional trigger to help seeking was concern that the implications of their experience might be more significant than their best initial hunch or lay diagnoses might suggest. 'Passing blood' (interview 11) was one of the commonest cited causes for concern:

'... actually I did have blood in my urine, which made me go to the doctor's 'cause I ... Yeah, I got a bit worried about that, certainly 'cause of my age ...' (interview 19: 143-144; aged 43 years)

'There was a lot of blood in my water and, um, I got a really big fright with that and that is what prompted me to go and see the doctor ... I just went, "ohhhh, there's got to be something wrong here"...' (interview 15: 6-12)

I'd had some bleeding as well, so I - I felt that it was a little more of a problem than could just be treated with - with fluids basically, so there was concern for that' (interview 6: 81-84)

Fear that infection might 'spread to the kidneys' also reportedly acted as a catalyst for help seeking:

'I'm always aware that it could spread to my kidneys and I could end up having kidney infections and feeling really desperate. So I decided to go to the doctor ...' (interview 9: 14-19)

'By the Monday the backache was quite nasty so I'm thinking "oh that's it I've got a kidney ... thingy bob".' (interview 1: 196-198)

Interviewee 1 also, unusually, expressed a fear about cancer, while another spoke in general terms about the fear caused by the severity of the pain:

'[l was in] terrible pain and [it was] frightening.' (interview 8: 86-87)

For another interviewee the recurring nature of her symptoms led her to worry about her health in general, and consequentially she sought reassurance:

'I had [an infection] back in February ... and I just got concerned that there may be something fundamentally wrong with my system that needed to be looked at ...' (interview 15: 75-77)

One interviewee alone expressed how her fear of worsening symptoms had prompted her to seek help in a preventative fashion. When asked by the interviewer whether her symptoms were severe, she responded thus:

'Well I was trying to catch them before they got too severe [exhaling/laughter] funnily enough!' (interview 7: 19-20)

\section{DISCUSSION}

\section{Summary of main findings and comparison with existing literature}

Prior to consulting, periods of self-care and watchful waiting were common and women's move to GP care should be viewed in this broader context. Levin broadly defined self-care as a 'decision-making process which involves self observation, symptom perception and labeling, judgment of severity, and choice and assessment of treatment options'. ${ }^{11}$ All of the interviewees had attempted self-care prior to visiting a GP (including an iterative period of monitoring and reevaluation). The present findings confirm Levin's description of self-care, ${ }^{11}$ and also resonate with 
McWhinney's observations that people may use selfcare to replace medical care and/or that self-care may precede consultation with the GP.12

Regardless of prior experience, women expressed fear of the consequences of continued symptoms particularly the potential for kidney infection. UTI was also perceived as alarming because of the nature of the actual already experienced symptoms. Blood in the urine triggered worry repeatedly and formed a strong driver for help seeking. In addition to specific fears, women reported that prior to consulting, the symptoms had an impact in a generalised or global way, affecting their mood and general overall healthiness. ${ }^{8}$ The search for reassurance, concern about complications, and worry about symptom duration were core drivers for consulting behaviour, and this has been shown in patients with other (often) self-limiting conditions, ${ }^{4,13}$ as has the highly evaluated nature of the decision to consult. ${ }^{14}$ This study highlights this strongly, and provides a clear reminder of the need to deal sensitively with the 'minor' illness of UTI, to acknowledge the 'primacy of the person', and attend to the unique experience of illness for each individual. ${ }^{12}$

It is noteworthy that in their interview accounts, interviewees did not discuss their lay referral and consulting networks as much as the study had anticipated, based on theories of health behaviour. ${ }^{15} \mathrm{It}$ may be that some interviewees believed that they were to blame for their UTI (for example, poor hygiene/drinking too much alcohol/caffeine) and this deterred them from speaking with others about it. ${ }^{16}$

\section{Strengths and limitations of the study}

Representation of thematic analysis can result in the decontextualisation of speakers' words. Care was therefore taken to analyse the interviewees' words in the broader context of the surrounding utterances.

Interviews provide useful perspectives on events or experiences but not a window to events as they occur. Moreover, interviewees will seek to manage the impression they make. In these interviews about health service use and self-care, it is unsurprising that a discursive appeal to being reasonable users of health services was often evident.

Interviewees came from a preselected population who had already consented to randomisation. It is, therefore, possible that a general practice population may not self-care to the extent reported in this study and this might threaten the transferability of these findings. Interviewers only spoke with women who had attempted to self-care but had still consulted their GP, thus excluding women who, for example, had either successfully self-cared or had not felt the need or desire to consult. ${ }^{12}$ However, similarities with other studies on the management of minor illness lend credibility/face validity to the present findings.

\section{Implications for future research and practice}

It is important that doctors are aware of patients' journeys prior to consulting and their reasons for doing so. Future research could usefully explore the drivers for symptomatic women not consulting.

\section{Funding body}

The Health Technology Assessment (HTA) programme funded the study. The HTA had no involvement in the research process or writing of this article $(97 / 14 / 06)$

\section{Ethics committee}

The study was approved by the Local Research Ethics Committee for Southampton and South West Hampshire (MREC/03/6/11)

\section{Competing interests}

The authors have stated that there are none

\section{Acknowledgements}

UTIS Team (in alphabetical order): Anne Arscott, Catherine Hawke, John A Lowes, Michael V Moore, Mark A Mullee, Kate Rumsby, David Turner and Greg Warner. We thank the practices who took part in the study and the women who gave their time and shared their experiences and thoughts.

\section{Discuss this article}

Contribute and read comments about this article on the Discussion Forum: http://www.rcgp.org.uk/bjgp-discuss

\section{REFERENCES}

1. Foxman B. Epidemiology of urinary tract infections: Incidence, morbidity, and economic costs. Am J Med 2002; 113(1): 5-13.

2. Office for Population Censuses and Surveys (OPCS). Morbidity statistics from general practice: fourth national study 1991. London: HMSO, 1994.

3. Gray NJ, Cantrill JA, Noyce PR. Health repertoires: an understanding of lay management of minor ailments. Patient Educ Couns 2002; 47(3): 237-244

4. Butler C, Rollnick S, Pill R, et al. Understanding the culture of prescribing: qualitative study of general practitioners' and patients' perceptions of antibiotics for sore throats. BMJ 1998; 317(7159): 637-642.

5. Everitt H, Kumar S, Little P. A qualitative study of patients' perceptions of acute infective conjunctivitis. Br J Gen Pract 2003; 53(486): 36-41.

6. Silverman D. Interpreting qualitative data: methods for analysing talk, text and interaction. 2nd edn. London: Sage Publications, 2001.

7. Barbour R and Members of WeReN. Acquiring qualitative skills for primary care research. Review and reflections on three-stage workshop. Part 2: analysing interview data. Fam Pract 2000; 17(1): 83-89.

8. Patton MQ. Qualitative research and evaluation methods. 3rd edn. London: Sage Publications, 2002.

9. Zola I. Pathways to the doctor: from person to patient. Soc Sci Med 1973; 7(9): 677-689.

10. Parsons T. The social system. London: Routledge and Kegan Paul Ltd, 1951

11. Levin L. Forces and issues in the survival of interest in self care: impetus for redirection in health. Health Educ Monogr 1977; 5(2): 115-121.

12. McWhinney I.R. A textbook of family medicine. 2nd edn. Oxford: Oxford University Press, 1997

13. Chan CSY. What do patients expect from consultations for upper respiratory tract infections? Fam Pract 1996; 13(3): 229-235.

14. Cantrill J, Morris C, Weiss M. How patients perceive minor illness and factors influencing seeing a doctor. Primary Health Care Research and Development 2006; 7(2): 157-164.

15. Freidson E. Client control and medical practice. Am J Sociol 1960; 65(4): 374-382.

16. Little P, Turner S, Rumsby K, et al. Dipsticks and diagnostic algorithm in urinary tract infection: development and validation, randomized trial, economic analysis, observational, cohort and qualitative study. Health Technol Assess 2009; 13(19): iii-iv, ix-xi, 1-73. 


\section{Appendix 1. Topic guide. Self-care and health-seeking} behaviour.

Please tell us about your recent experience of urinary infection/inflammation

Topics to cover: Self-care/triggers/symptom recognition/lay diagnosis

Areas probed:

- Signs/symptoms?

- Severity?

- Duration?

- Self-help/care tried prior to GP visit? If yes what? For example, potassium citrate/discussion with pharmacist? If not, why?

- Previous experience of UTIs?

- Previous experience of seeing doctor and getting treatment?

- Discussed with/consulted family/friend(s)/partner?

- Factors that shaped decision to attend GP on this occasion? 